\title{
Impacto da Nefrite sobre os Resultados Gestacionais de Mulheres com Lúpus Eritematoso Sistêmico(*)
}

\section{The Impact of Nephritis on Gestational Results in Women with Systemic Lupus Erythematosus}

\author{
Evandro M. Klumb ${ }^{(1)}$, Laura M. S. Barros ${ }^{(2)}$, Leonardo Romeiro ${ }^{(3)}$, Nilson R. de Jesús ${ }^{(4)}$, \\ Roger A. Levy ${ }^{(5)}$, Elisa M. N. Albuquerque ${ }^{(6)}$
}

\section{RESUMO}

Objetivo: avaliar o impacto da nefrite lúpica e suas complicações nos resultados gestacionais. Métodos: avaliamos retrospectivamente 76 gestações em 63 pacientes com lúpus eritematoso sistêmico (LES). Resultados: a hipertensão arterial estava presente como complicação clínica em 23 (30\%) gestações. Vinte e sete (36\%) gestações ocorreram em 19 pacientes com nefrite. Encontramos um número significativamente mais elevado de óbitos fetais quando avaliamos todas as pacientes com nefrite em comparação com aquelas sem nefrite (37\% e 12,2\%, respectivamente $[p=0,019])$. Conclusões: além da atividade de nefrite e do diagnóstico de nefrite propriamente dito, tiveram relação com pior sobrevida fetal a associação da síndrome do anticorpo antifosfolipídeo ou detecção de um dos anticorpos relacionados, a presença HAS e insuficiência renal (mesmo em suas fases iniciais).

Palavras-chave: gestação no lúpus, pré-eclampsia, nefrite, antifosfolipídeo.

\section{INTRODUÇÃO}

Na década de 1950, a gravidez ocorria somente em uma paciente entre 1.500 portadoras de lúpus eritematoso sistêmico (LES) e essa era aparentemente relacionada com exa-

\begin{abstract}
Objective: To evaluate the impact of systemic lupus erythematosus (SLE) nephritis and its complications on the gestational results. Methods: We evaluated retrospectively 76 pregnancies in 63 SLE patients. Results: Arterial hypertension was detected as a clinical complication in $23(30 \%)$ pregnancies. Twentyseven (36\%) pregnancies occurred in 19 patients with lupus nephritis. We found a significantly increased number of fetal deaths when patients with nephritis were compared with those without nephritis (37\% and $12.2 \%$ respectively, $p=0.019$ ). Conclusions: In addition to active nephritis and its diagnosis, there was a worse fetal survival rate when there was an association with antiphospholipid syndrome or one of the antibodies related to it, the presence of arterial hypertension and renal failure (even in early stages).
\end{abstract}

Keywords: lupus pregnancy, preeclampsia, nephritis, antiphospholipid.

cerbações da doença, morte fetal e até morte materna ${ }^{(1)}$. Nos últimos vinte anos, o melhor entendimento dos aspectos imunológicos da doença favoreceu um melhor tratamento, que resultou no aumento da sobrevida e da qualidade de vida das pacientes, refletindo em incremento na

\footnotetext{
* Trabalho realizado na Disciplina de Reumatologia, Faculdade de Ciências Médicas, Universidade do Estado do Rio de Janeiro (FCM-UERJ), Rio de Janeiro, RJ, Brasil. Recebido em 17/01/2005. Aprovado, após revisão, em 04/04/2005.

1. Mestre em Medicina, reumatologista. Disciplina de Reumatologia, FCM-UERJ.

2. Residente. Disciplina de Obstetrícia, FCM-UERJ.

3. Residente. Disciplina de Reumatologia, FCM-UERJ.

4. Professor assistente. Disciplina de Obstetrícia, FCM-UERJ.

5. Professor adjunto. Disciplina de Reumatologia, FCM-UERJ.

6. Professora assistente. Disciplina de Reumatologia, FCM-UERJ.
}

Endereço para correspondência: Evandro M. Klumb. Blvd. 28 de setembro, 77, sala 333, Vila Isabel. Rio de Janeiro, RJ, Brasil. Tel. (21) 2587-6316; e-mail: eklumb@uol.com.br 
possibilidade de tornarem-se grávidas, desde que a fertilidade da maioria das pacientes com LES não está prejudicada $^{(2,3)}$.

Com a identificação da síndrome do anticorpo antifosfolipídeo (SAF) no final da década de 1980, tornou-se evidente que as perdas fetais recorrentes, e outras complicações trombóticas, são causadas pela SAF e não uma "atividade" do $\operatorname{LES}^{(4)}$. Várias das manifestações anteriormente atribuídas à atividade do LES foram posteriormente identificadas como decorrentes da gestação por si só (como elevação da VHS, cloasma gravídico, dispnéia e gonalgia) ou de complicações próprias da gestação (como anemia, pré-eclâmpsia e eclâmpsia). O comprometimento da função renal decorrente do LES ou da SAF, quando em fase ativa na concepção, pode realmente se agravar durante a gestação, momento no qual o arsenal terapêutico é limitado. Nem sempre é fácil diferenciar atividade renal relacionada ao LES, pré-eclâmpsia superposta em paciente com ou sem nefrite e complicações da microangiopatia relacionada à presença de anticorpos antifosfolipídeos ${ }^{(5-7)}$. Segue-se a recomendação de que a época ideal para engravidar é após três meses sem proteinúria $(<300 \mathrm{mg} / 24 \mathrm{~h})$ e com sedimento urinário inocente ${ }^{(8)}$. O impacto da atividade do LES durante a gestação nos resultados gestacionais ainda é assunto de debate. Alguns relatos indicam que a atividade, principalmente renal, poderia estar relacionada com aumento de perdas fetais e outras complicações obstétricas ${ }^{(9-10)}$. Outros, mais detalhados, não relacionam a atividade renal com perda fetal, mas somente com prematuridade, ruptura prematura das membranas ovulares e hipertensão $\operatorname{arterial}^{(11-13)}$. Atualmente é evidente que o maior risco de perda fetal tem relação com a SAF ${ }^{(14-16)}$.

Em uma análise retrospectiva de uma coorte de lúpus de 74 gestações, publicada em 1992, foi notada uma correlação da atividade do LES com perda fetal. Houve, no entanto, uma relação ainda mais marcante com prematuridade e outras complicações gestacionais $^{(17)}$. Um estudo mais recente do mesmo grupo confirmou essas observações e recomenda que as pacientes com nefrite devam manter o uso de hidroxicloroquina e azatioprina durante a gestação ${ }^{(13)}$. O efeito da nefrite lúpica nos resultados gestacionais foi analisado em dois estudos e a presença de anticorpos antifosfolipídeos (aPL) foi o principal determinante de perda fetal. Packham et al. estudaram 41 gestantes com nefrite: houve 53\% de perda fetal com aPL e $14 \%$ de perda nas pacientes sem aPL ${ }^{(18)}$. No estudo de Moroni et al., nas 48 gestantes com nefrite, houve $83 \%$ de perda fetal com aPL e $13 \%$ de perda nas com aPL negativo $^{(19)}$.
O presente estudo teve por objetivo principal analisar as repercussões da coexistência de nefrite lúpica sobre o binômio materno fetal.

\section{PACIENTES E MÉTODOS}

Neste estudo, realizamos uma avaliação retrospectiva dos dados clínicos e laboratoriais referentes a 76 gestações, obtidos dos prontuários de 63 pacientes com LES, cujos acompanhamento pré-natal e parto foram realizados no Hospital Universitário Pedro Ernesto, da Universidade do Estado do Rio de Janeiro (HUPE/UERJ). Foram selecionadas para análise as pacientes acompanhadas entre janeiro de 1996 e dezembro de 2004, que preenchiam os critérios para classificação de LES de acordo com o Colégio Americano de Reumatologia ${ }^{(20)}$.

Para as pacientes que apresentavam diagnóstico de nefrite antes do início da gravidez (OMS classes III, IV, V ou síndrome nefrótica na ausência de biópsia renal), também foram avaliadas a evolução da proteinúria, função renal e desenvolvimento de alterações do sedimento urinário. Neste estudo estabelecemos como critério de ativação/reativação renal o desenvolvimento de dois ou três dos critérios abaixo:

Critério A - Elevação de creatinina: quando houve elevação da creatinina maior ou igual a $25 \%$ do valor anterior à gravidez.

Critério B - Elevação da proteinúria quando:

- proteinúria $\geq 1000 \mathrm{mg}$ sendo que a basal era inferior a 500 mg; ou

- proteinúria $\geq 2000 \mathrm{mg}$ sendo que a basal era $\geq 500$ mg e < 1000 mg; ou

- houve duplicação do valor da proteinúria e o pré-gravídico era $\geq 1000 \mathrm{mg}$.

Critério C - Atividade no sedimento urinário: quando houve duas das seguintes alterações: desenvolvimento de hematúria, leucocitúria, cilindros celulares ou presença de mais de 50\% dos eritrócitos com dismorfismo de padrão glomerular.

Obtivemos dados referentes às características clínicas das pacientes, tais como intercorrências de hipertensão arterial sistêmica (HAS), nefrite, presença de aPL, necessidade de elevação da dose de corticóide e/ou imunossupressor durante a gestação; além dos dados referentes à vitalidade do concepto (abortamento, idade gestacional ao parto, peso, nati e neomortalidade, necessidade de internação em unidade de terapia intensiva neonatal e uso de prótese ventilatória), e dados referentes à evolução materna, in- 
cluindo desenvolvimento de eclâmpsia (ECL)/pré-eclâmpsia (PRE-ECL) e ruptura prematura das membranas ovulares (RPMO).

Como todos os desfechos foram binomiais e houve violação do pressuposto de independência entre as observações (nove pacientes apresentaram mais de uma gestação), usamos o modelo de equações de estimação generalizadas com função de ligação logit. Estimamos as razões de chance para cada variável preditora (nefrite, HAS, presença do aPL e tempo de LES ) para os desfechos de sucesso de gestação, definidos neste estudo como: natimortalidade, neomortalidade (óbito até 28 dias de vida), total de óbitos, préeclâmpsia/eclâmpsia, RPMO e necessidade de internação em unidade de terapia intensiva neonatal com os respectivos intervalos de confiança de 95\%. Estabelecemos em 0,05 o índice de significância estatística para os resultados obtidos.

O teste exato de Fisher foi utilizado para comparação de variáveis categóricas quando indicado.

\section{RESULTADOS}

Sessenta e três pacientes portadoras de LES tiveram 76 gestações espontâneas. Cinqüenta e três (85\%) eram gestantes pela primeira vez, cinco (8\%) pela segunda, três (5\%) pela terceira e uma (2\%) pela quarta. A idade média das pacientes ao iniciar a gravidez foi de 27 anos, variando de 16 a 44 anos. O tempo médio entre o início da doença e a concepção foi de seis anos, variando desde a eclosão durante a gravidez (um caso) até 24 anos. A HAS esteve presente como complicação clínica em 23 (30\%) gestações. Vinte e seis gestações (34\%) ocorreram em pacientes que preencheram os critérios propostos para a classificação com nefrite com diagnóstico anterior à gravidez. Em uma paciente a nefrite surgiu pela primeira vez, durante a gestação. Nas Tabelas 1 e 2 estão descritas as características das pacientes estudadas.

Este estudo não teve como objetivo determinar a influência da gestação sobre a atividade da doença, pois foi realizado com análise retrospectiva, o que torna impraticável o estabelecimento confiável de índices como o SLEDAI ou mais especificamente o SLEPDAI. No entanto, observamos que a maior parte das nossas pacientes iniciou a gestação sem sinais de atividade da doença (70 de 74 gestações) e que $42 \%$ dessas estavam em uso de corticosteróide. Identificamos 14 pacientes que tiveram necessidade de elevação da dose de corticosteróide determinada durante o atendimento no período pré-natal, por avaliação clínica de um reumatologista em conjunto com o obstetra. Em três casos,
TABELA 1

CARACTERÍ́sticas das 76 gestaÇÕes EM 63 PaCientes

\begin{tabular}{|c|c|c|}
\hline Características & \multicolumn{2}{|c|}{ Valor médio (variação) } \\
\hline Idade - anos & \multicolumn{2}{|c|}{$27(16-44)$} \\
\hline Tempo LES - Gestação - anos & \multicolumn{2}{|r|}{$6(0-24)$} \\
\hline Medicação & \multicolumn{2}{|r|}{ N (\%) } \\
\hline Prednisona & \multicolumn{2}{|r|}{$32(42 \%)$} \\
\hline Azatioprina & \multicolumn{2}{|r|}{$17(22 \%)$} \\
\hline Intercorrências & \multicolumn{2}{|r|}{ N (\%) } \\
\hline $\mathrm{aPL} L^{*}$ & \multicolumn{2}{|r|}{$26(47 \%)$} \\
\hline HAS & \multicolumn{2}{|r|}{$19(25 \%)$} \\
\hline Nefrite & \multicolumn{2}{|r|}{$27(35 \%)$} \\
\hline \multicolumn{3}{|c|}{ * Testes laboratoriais realizados em 55 gestações } \\
\hline \multicolumn{3}{|c|}{ TABELA 2} \\
\hline \multicolumn{3}{|c|}{$\begin{array}{l}\text { CARACTERÍSTICAS GERAIS DAS PACIENTES COM E } \\
\text { SEM NEFRITE EM } 76 \text { GESTAÇÕES }\end{array}$} \\
\hline & $\begin{array}{c}\text { Com nefrite } \\
n=27\end{array}$ & $\begin{array}{l}\text { Sem nefrite } \\
n=49\end{array}$ \\
\hline Idade em anos - média ( \pm DP) & $28( \pm 5,05)$ & $27,1( \pm 5,4)$ \\
\hline HAS. n (\%) & $21(77,7 \%)$ & $2(0,5 \%)$ \\
\hline aPL. $n(\%)$ & $13 / 22(59 \%)$ & $15 / 36(42 \%)$ \\
\hline Tempo de LES em anos ( \pm DP) & $8,05( \pm 7,05)$ & $5,47( \pm 10,3)$ \\
\hline
\end{tabular}

a elevação decorreu de atividade renal do lúpus. Dezessete gestações $(22 \%)$ se iniciaram durante o uso de azatioprina.

No que se refere ao resultado gestacional das 76 gestações estudadas, 11 (15\%) desenvolveram pré-eclâmpsia e destas 54\% tinham HAS e nefrite. A RPMO ocorreu em 27\% $(n=20)$ dos casos e foi responsável por $40 \%(n=8)$ dos nascimentos prematuros. A incidência de $10 \%$ de abortamentos está, provavelmente, influenciada pela não inclusão no prénatal das pacientes com perda gestacional precoce. Das gestações que ultrapassaram a $20^{\mathrm{a}}$ semana, 7 (9,5\%) resultaram em natimortos e $5(7,4 \%)$ em neomortos, o que correspondeu à taxa de mortalidade perinatal de 162\%o. A HAS (OR $=4,74-$ IC95\% - 1,30-17,23) e a nefrite $(\mathrm{OR}=4,12$ - IC95\% - 1,27-13,39) apresentaram associação significativa com a perda perinatal. O sofrimento fetal crônico foi diagnosticado em 21 (27,6\%) gestações, por meio da alteração de pelo menos uma avaliação biofísica fetal (restrição do crescimento, oligodramnia, Doppler velocimetria e cardiotocografia). A idade gestacional média ao nascer foi de 35 semanas com variação 24 a 42 semanas. O parto ocorreu antes da $37^{\mathrm{a}}$ semana para 30 (44\%) dos 68 nascidos vivos. Quatorze (20,6\%) recém-nascidos apre- 
sentavam restrição do crescimento e a presença de aPL foi a intercorrência que apresentou maior associação com o crescimento intrauterino restrito (CIUR) $(\mathrm{OR}=10,20-$ IC95\% - 2,76 - 37,63).

No subgrupo com LES e nefrite, analisamos a evolução de 27 gestações em 18 pacientes que haviam desenvolvido glomerulonefrite antes de engravidar e uma gestação em paciente que desenvolveu nefrite na $24^{\mathrm{a}}$ semana durante o acompanhamento pré-natal. A classe IV, de acordo com a classificação OMS, foi a mais freqüente (12 pacientes). Quatro gestações ocorreram em mulheres que apresentaram síndrome nefrótica sem que tenham sido submetidas à biópsia renal; duas apresentavam glomerulonefrite focal; duas, membranosa; e uma, associação das classes IV e V. Onze pacientes apresentavam concomitantemente algum tipo de aPL (anticorpos anticardiolipina (títulos moderados ou altos) e/ou anticoagulante lúpico). Dessas seis, quatro foram submetidas à biópsia renal, que evidenciou em duas glomerulonefrite lúpica (OMS - classes III e V) e duas pacientes (não biopsiadas) apresentaram síndrome nefrótica associada à hematúria dismórfica e cilindrúria no sedimento urinário, razão pela qual foram classificadas como portadoras de nefrite lúpica, e não como manifestações renais da SAF (Tabela 3) ${ }^{(21-22)}$.

No grupo total de pacientes com 76 gestações, observamos 16 óbitos (21\%) dos conceptos. A análise das gestações cujas mães não apresentavam previamente envolvimento renal (49 gestações), evidenciou morte intra-uterina e neonatal em 12,2\% dos casos e dentre as 27 gestações em 19 pacientes com NL, houve 10 óbitos fetais (37\%), sendo 6 natimortos e 4 mortes em até 28 dias do parto (neomortos), com diferença entre os grupos estatisticamente significativa $(p=0,019)$ (Figura 1). Ao analisarmos apenas o grupo com GN sem AC antifosfolipídios, com 14 gestações, encontramos quatro óbitos fetais $(28,6 \%)$, todos natimortos (Tabelas 2, 4 e 5). Essa diferença, no entanto, não foi estatisticamente significativa $(p=0,6)$. Entre todas as variáveis analisadas, apenas a HAS teve associação positiva com a morte neonatal ou natimortalidade $(\mathrm{p}=0,018)$.

Durante a gravidez, três pacientes apresentaram reativação renal, de acordo com o critério proposto, com evolução para o óbito fetal em duas (uma morte do feto intraútero, uma após 30 horas do nascimento) e um caso teve boa evolução com índices Apgar 8 e 9 no $1^{\circ}$ e $5^{\circ}$ minutos respectivamente (Tabela 6 ). $O$ tratamento nesses casos incluiu aumento da dose de prednisona e introdução de azatioprina. Nos pacientes com nefrite, encontramos cinco casos de pré-eclâmpsia, todos em pacientes que apresentavam anticorpos aPL (Tabela 2).
TABELA 3

CARACTERÍSTICAS DAS PACIENTES COM NEFRITE EM 27 GESTAÇÕES

\begin{tabular}{lc}
\hline Creatinina inicial & $1,25( \pm 1,1)$ \\
\hline Creatinina final & $1,30( \pm 1,7)$ \\
OMS - classe histológica & \\
III - & 02 \\
IV - & 13 \\
V - & 02 \\
Síndrome nefrótica (sem biópsia) & 04 \\
\hline
\end{tabular}

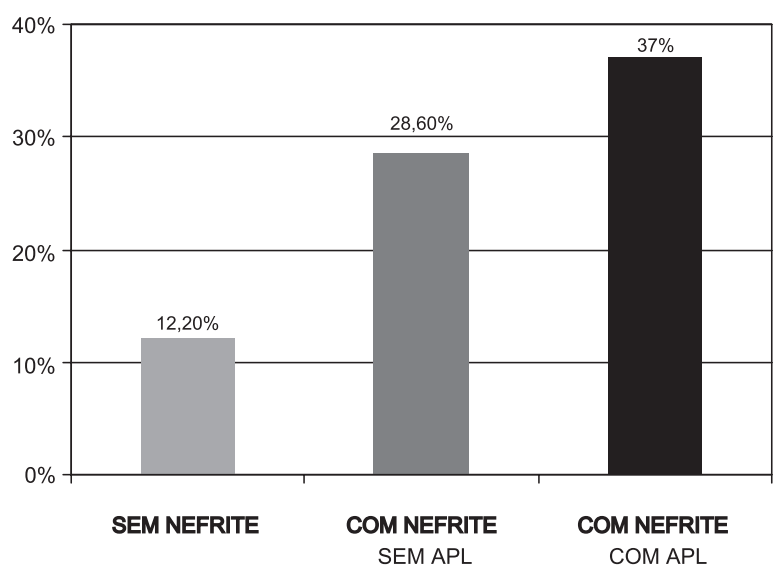

${ }^{*} p=0,019$ (equações de estimação generalizadas).

Figura 1 - Total de perdas (nati e neomortalidade) em pacientes com e sem nefrite em 76 gestações de pacientes com LES

TABELA 4

GESTAÇÕES EM PACIENTES COM LÚPUS - FATORES RELACIONADOS AO RESULTADO GESTACIONAL ADVERSO $(\mathrm{N}=76)$.

NATI E NEOMORTALIDAdE

\begin{tabular}{lcc}
\hline & $\begin{array}{c}\text { Natimortos } \\
\mathbf{n}=\mathbf{9}\end{array}$ & $\begin{array}{c}\text { Neomortos } \\
\mathbf{n}=5\end{array}$ \\
\hline Nefrite $-\mathrm{n} ; \mathrm{p}$ & $8 ; \mathrm{p}=0,007^{*}$ & $2 ; \mathrm{p}=0,593^{*}$ \\
HAS $-\mathrm{n} ; \mathrm{p}$ & $6 ; \mathrm{p}=0,007^{*}$ & $1 ; \mathrm{p}=0,725^{*}$ \\
AC anti fosfolipídio $n ; p$ & $4 ; p=0,318^{* *}$ & $2 ; p=0,208^{*}$ \\
\hline
\end{tabular}

* equações de estimação generalizadas; ** teste exato de Fisher

TABELA 5

GESTACÕES EM PACIENTES COM LÚPUS - FATORES RELACIONADOS AO Resultado gestacional adVErso $(\mathrm{N}=76)$. Total de Óbitos

\begin{tabular}{lcccc}
\hline & $\mathbf{n}$ & $\mathbf{p}$ & $\mathbf{0 R}$ & IC 95\% \\
\hline Nefrite & 10 & $0,019^{*}$ & 4,12 & $1,27-13,39$ \\
HAS & 07 & $0,018^{*}$ & 4,12 & $1,30-17,23$ \\
AC anti fosfolipídio & 06 & $0,60 * *$ & & \\
\hline * equações de estimação generalizadas; ** teste exato de Fisher &
\end{tabular}


TABELA 6

AtivaÇÃo DE NeFrite LÚPICA DURANTE A GESTAÇÃO COM MORTE FETAL $(\mathrm{N}=2)$

\begin{tabular}{|c|c|c|}
\hline & Natimorto $(n=1)$ & Neomorto $(n=1)$ \\
\hline Critério A - Elevação da creatinina & Elevação da creatinina de $1,2 \mathrm{mg} / \mathrm{dL}$ para $1,7 \mathrm{mg} / \mathrm{dL}$ & Elevação da creatinina de $1,1 \mathrm{mg} / \mathrm{dL}$ para $1,6 \mathrm{mg} / \mathrm{dL}$ \\
\hline Critério B - Aumento da proteinúria & Elevação da proteinúria de $2,4 \mathrm{~g} / 24 \mathrm{~h}$ para $7 \mathrm{~g} / 24 \mathrm{~h}$ & Elevação da proteinúria de $1,8 \mathrm{~g} / 24 \mathrm{~h}$ para $4 \mathrm{~g} / 24 \mathrm{~h}$ \\
\hline Critério C - Sedimento urinário ativo & $\begin{array}{l}410.000 \text { hemácias } / \mathrm{mL} \text {. } \\
\text { Cilindros com inclusões hemáticas. } \\
\text { Dismorfismo de padrão glomerular em } \\
85 \% \text { das hemácias }\end{array}$ & $\begin{array}{l}280.000 \text { hemácias } / \mathrm{mL} \text {. } \\
\text { Cilindros com inclusões leucocitárias. } \\
\text { Dismorfismo de padrão glomerular em } 80 \% \text { das } \\
\text { hemácias }\end{array}$ \\
\hline
\end{tabular}

\section{DISCUSSÃO}

Neste estudo, realizado retrospectivamente em uma unidade de referência para gestação de alto risco, onde todas as consultas de pré-natal e acompanhamento hospitalar são feitos em conjunto por obstetras e reumatologistas, procuramos identificar os fatores que se relacionaram ao sucesso da gestação, em especial em um subgrupo de mulheres que apresentavam nefrite lúpica OMS classes III, IV, V ou síndrome nefrótica sem biópsia. Não tivemos a preocupação de estabelecer a influência da gestação como elemento determinante de atividade do LES, nem mesmo a melhor alternativa terapêutica para os problemas identificados ao longo das gestações. Analisamos cada gestação como um evento isolado, à semelhança da maioria dos estudos publi$\operatorname{cados}^{(23)}$, apesar de reconhecermos que isso pode determinar um viés quanto à efetiva independência de cada gravidez em face dos desfechos de análise. Realizamos uma análise univariável para nefropatia e HAS em relação aos desfechos estudados, em decorrência do alto grau de associação entre essas variáveis, demonstrada através do cálculo do $\varphi$ de Cramer ${ }^{(24)}$.

O total de abortamentos identificado em nossa série foi de 10\%, inferior ao relatado na maioria dos estudos. Este número pode estar subestimado, uma vez que o nosso banco de dados para análise originou-se no acompanhamento pré-natal, sendo possível que algumas pacientes tenham tido abortamentos sem que estes tenham sido documentados no banco. O critério que estabelecemos para determinação de atividade de GN foi semelhante ao proposto por Sidiropoulos et al. ${ }^{(25)}$, contudo, definimos valores específicos para a elevação da creatinina (em 20\%) e proteinúria, tendo em vista que na gravidez normal existe habitualmente redução da primeira e elevação da segunda apenas pelas alterações hemodinâmicas próprias dessa fase. Identificamos três pacientes (11\%) que desenvolveram atividade (nefrítica) da nefrite lúpica, sendo que em uma, essa foi manifestação de abertura da doença. A maioria das séries publicadas de gestação em pacientes com nefrite lúpica tem número de casos semelhante ao estudado por nós e identificou reativação renal em $12 \% \%^{(25)}$ a $42,9 \%{ }^{(26)}$ dos pacientes, sendo essas mais freqüentes quando a gravidez se desenvolveu quando a doença encontrava-se ativa. Por outro lado, a utilização de diferentes critérios para caracterização de atividade geral e renal entre os diferentes estudos dificulta a comparação entre essas séries e talvez tenha contribuído para a discrepância entre os resultados encontrados nos diferentes estudos. Em outras quatro pacientes, observamos flutuações dos níveis de proteinúria, sem elevações concomitantes da creatinina sérica ou alterações significativas no sedimento urinário, razão de não termos classificado esses casos como reativação renal.

Cinco pacientes com nefrite desenvolveram PRE-ECL (5/27 - 18,7\%), em comparação com seis casos (6/47 $12,7 \%)$ das que não apresentavam envolvimento renal prévio, com diferença estatisticamente significante $(p=0,047)$, resultado semelhante ao descrito por outros autores $(14,3 \%)^{(26)}$. Não encontramos em nossa série associação entre a presença de aPL e PRE-ECL $(\mathrm{p}=0,43)$, possivelmente em decorrência do pequeno número de eventos e ausência de pesquisa dos aPL em todos os casos. Em relação à diferenciação entre a flutuação da proteinúria, desenvolvimento de edema e mesmo caracterização do diagnóstico de PRE-ECL em pacientes com nefrite lúpica no momento da concepção. Diversos autores têm valorizado as alterações no sedimento urinário como o marcador mais fidedigno nesta diferenciação( ${ }^{(27)}$, uma vez que apenas raramente indicamos a biópsia renal nessa fase. No acompanhamento de nossos pacientes, entendemos de particular utilidade nesse diagnóstico diferencial, a presença na urina de cilindros celulares, mormente os com inclusões hemáticas e/ou a presença de dismorfismo eritrocitário de padrão glome$\operatorname{rular}^{(29)}$, em porcentual superior a $50 \%{ }^{(25)}$, apesar de desco- 
nhecermos estudos com metodologia adequada que permitam estabelecer essa afirmativa como evidência científica.

Encontramos em nosso estudo um número elevado de óbitos fetais ao avaliarmos todas as pacientes com nefrite: $37 \%$. Este valor é superior ao descrito por outros autores em séries com pacientes com nefrite ${ }^{(23,30)}$; é, contudo, semelhante ao reportado em alguns estudos, cujos porcentuais de perdas fetais variam de $14 \%$ a $68 \%{ }^{(30)}$. Em contraste com os casos acompanhados por Huong et al. ${ }^{(30)}$ e Cervera et al. ${ }^{(23)}$, os nossos pacientes com nefrite apresentavam freqüência elevada de hipertensão arterial 21/27 (77\%), o que pode ter contribuído como fator determinante para o elevado número de resultados gestacionais adversos encontrados. Quando realizamos o cálculo do $\varphi$ de Cramer, observamos que havia uma elevada associação entre HAS e nefropatia, o que tornou impossível estabelecer qual dos dois fatores contribuiu, ou se os dois contribuíram igualmente para o resultado gestacional adverso.

Por outro lado, ao estratificarmos o grupo de gestantes com nefrite, excluindo as que apresentavam aPL, observamos que a mortalidade fetal foi de $28,6 \%$, e de $46 \%$ quando analisadas apenas as pacientes com nefrite e aPL, reforçando o elevado efeito negativo dos aPL sobre a evo-

\section{REFERÊNCIAS}

1. Jesús NR, Chaves Neto H, Montenegro CAB: Lúpus eritematoso sistêmico - Repercussões sobre o concepto. J Bras Ginec 99: 14350,1989

2. Cervera R, Font J, Carmona F, Balasch J: Pregnancy outcome in systemic lupus erythematosus: good news for the new millennium. Autoimmunity Rev 1: 354-9,2002.

3. Pavão ALB, Klumb EM, Suassuna JHR, Pacheco MS, Souza PFM, Albuquerque EMN: Sobrevida em 5 e 10 anos em 78 pacientes com nefrite lúpica. Rev Bras Reumatol 44: S198, 2004.

4. Hughes GRV, Harris EN, Gharavi AE: The anticardiolipin syndrome. J Rheum 13: 486-9,1986.

5. Branch DW, Andres R, Digre KB, Rote NS, Scott JR: The association of antiphospholipid antibodies with severe pre-eclampsia. Obstet Gynecol 73: 541-5, 1989.

6. Burkett G: Lupus nephropathy and pregnancy. Clin Obstet Gynecol 28: 310-23, 1985.

7. Kincaid-Smith PS, Fairley KF, Kloss M: Lupus anticoagulant associated with renal thrombotic microangiopathy and pregnancyrelated renal failure. Q J Med 69: 795-815,1988.

8. Moroni G, Ponticelli C: The risk of pregnancy in patients with lupus nephritis. J Nephrol 16: 161-7, 2003.

9. Imbasciati E, Surian M, Bottino S, et al: Lupus nephropathy and pregnancy. A study of 26 pregnancies in patients with systemic lupus erythematosus and nephritis. Nephron 36: 46-51,1984.

10. Hayslett JP, Lynn RI: Effect of pregnancy in patients with lupus nephropathy. Kidney Int 18: 207-20,1980. lução da gestação, apesar de não termos encontrado significância estatística nessa diferença. Por ser um estudo retrospectivo, não obtivemos a análise histopatológica da placenta de todos os casos, mas em alguns ficou evidente a participação de eventos vaso-oclusivos placentários na determinação do sofrimento e morte fetal.

Além do papel da associação da SAF ou apenas dos aPL, e da existência de atividade da doença no momento do início da gestação, também teve importância capital na sobrevida fetal a presença HAS e insuficiência renal (mesmo em suas fases iniciais). Em nossa série, apenas seis pacientes não apresentavam HAS, ao passo que somente uma tinha níveis séricos de creatinina acima de $1,5 \mathrm{mg} / \mathrm{dL}$, que foi relacionada de forma significativa com o risco de perda fetal por outros autores ${ }^{(32)}$. Em nosso estudo encontramos níveis elevados de óbito fetal, superiores aos descritos por estudos prospectivos. Entendemos que esta diferença seja decorrente da maior seleção de pacientes estáveis nessas séries. Os pacientes por nós acompanhados se assemelham mais intensamente aos encontrados na prática clínica com mulheres que concebem em uso de ciclofosfamida, em franca atividade de doença, com aPL, com doses elevadas de corticosteróides e outros comprometimentos mais graves.

11. Lockshin MD, Reinitz E, Druzin ML, Murrman M, Estes D: Lupus pregnancy: case-control study demonstrating absence of lupus exacerbation during or after pregnancy. Am J Med 77: 893-8,1984.

12. Cortes-Hernández J, Ordi-Ros J, Paredes F, Casellas M, Castillo F, Vilardell-Tarres M: Clinical predictors of fetal and maternal outcome in systemic lupus erythematosus: a prospective study of 103 pregnancies. Rheumatology 41: 643-50, 2002.

13. Clowse MEB, Magder LS, Witter F, Petri M: The impact of increased lupus activity on obstetric outcome. Arthritis Rheum 52: 514-21, 2005.

14. Lockshin MD, Druzin ML, Goei S, et al: Antibody to cardiolipin as a predictor of fetal distress or death in pregnant patients with systemic lupus erythematosous. N Engl J Med 313: 152-6,1985.

15. Pattison NS, Chamley LW, McKay EJ, Liggins GC, Butler WS: Antiphospholipid antibodies in pregnancy: prevalence and clinical associations. Br J Obstet Gynaecol 100: 909-13,1993.

16. Loizou S, Byron MA, Englert HJ, David J, Hughes GR, Walport MJ: Association of quantitative anticardiolipin antibody levels with fetal loss and time of loss in systemic lupus erythematosus. Q J Med 68: 525-31,1988.

17. Petri M, Howard D, Repke J: Frequency of lupus flare in pregnancy. The Hopkins Lupus Pregnancy Center experience. Arthritis Rheum 34: 1538-45,1991.

18. Packham DK, Lam SS, Nicholls K, Fairley KF, Kincaid-Smith PS: Lupus nephritis and pregnancy. Q J Med 83: 315-24,1992.

19. Moroni G, Quaglini S, Banfi G, et al: Pregnancy in lupus nephritis. Am J Kidney Dis 40: 713-20,2002. 
20. Tan EM, Cohen AS, Fries JF, et al: Criteria for the classifications of systemic lupus erythematosus. Arthritis Rheum 25: 53,1982.

21. Nochy D, Daugas E, Droz D, et al: The intrarenal vascular lesions associated with primary antiphospholipid syndrome. J Am Soc Nephrol. 10: 507-18, 1999.

22. D'Cruz D: Renal manifestations of antiphospholipid syndrome. Lupus 14: 45-8, 2005.

23. Cervera R, Font J, Carmona F, Balasch J: Pregnancy outcome in systemic lupus erythematosus: Good news for the millennium. Autoimmunity Rev 1: 354-59, 2002.

24. Liang K, Zeger SL: Longitudinal data analysis using generalized linear models. Biometrika 73: 13-22, 1986.

25. Sidiropoulos PI, Kritikos HS, Boumpas DT: Lupus nephritis flare. Lupus 14:49-52, 2005.

26. Tincani A, Faden D, Tarantini N, et al: Systemic lupus erythematosus and pregnancy: a prospective study. Clin Exp Rheumatol 10: 439-46, 1992.
27. Ramos A, Veloz Z, Medina F, et al. The outcome of lupus nephritis and pregnancy. Arthritis Rheum 42; S214, 1999.

28. Gordon C: Pregnancy and autoimmune diseases. Best Pract Res Clin Rheum 18: 359-79, 2004.

29. Tomita M, Kitamotoy, Nakayama M, Sato T: A new morphologic classification of urinary erythrocytes for differential diagnosis of glomerular hematuria. Clin Nephrol 37: 84-9, 1992.

30. Huong DLT, Wechsler B, Vauthier-Brouzes D, Beaufils H, Lefebvre G, Piette JC: Pregnancy in past or present lupus nephritis: a study of 32 pregnancies from a single center. Ann Rheum Dis 60: 599-604, 2001.

31. Kitridou RC, Goodwin TM: The fetus in systemic lupus erythematosus. In: Dubois' lupus erythematosus. 6th ed. Philadelphia, Lippincott Williams \& Wilkins, 2002. 